\title{
The comparative evaluation of uterine sarcoma:
} \section{preoperatively unexpected or diagnosed? Does it}

\section{matter?}

\author{
Cetin Kılıççı \\ Zeynep Kamil Maternity and Children's Training and Research Hospital, Department of Obstetrics and Gynecology
}

\begin{abstract}
We aim to identify clinical characteristics, outcome and prognosis of uterine sarcoma cases and compare clinical outcome of preoperatively or postoperatively (unexpected) diagnosed patients.

In this retrospective study, medical records of patients with uterine sarcoma were evaluated at the Zeynep Kamil Women and Children Education and Research Hospital, Department of Obstetrics and Gynecology, from January 2009 to December 2016. The age at diagnosis, gravidity, parity, menopausal status, complaints, comorbidity, tumor grade, tumor histology, stage of disease, postoperative adjuvant therapy, recurrence rate, location of recurrence, follow-up interval, and survival rate were analysed. Additionally, patients were divided into two groups: patients with diagnosed preoperatively and diagnosed postoperatively (unexpected). Two groups were compared regarding clinical characteristics.

Sixty-three patients were found to have uterine sarcoma. Mean age of patients was $51.8 \pm 11.7$. Twenty-nine (46\%) patients had leiomyosarcoma, $11(17.5 \%)$ patients had endometrial stromal sarcoma and $13(20.6 \%)$ had malign mixt mullerian tumor. Of 63 patients, 49 had pre-operative uterine sarcoma diagnosis and 14 patients had unexpected uterine sarcoma. The incidence of unexpected sarcoma was detected as $0.3 \%$. The recurrence rate was significantly higher in patients with unexpected uterine sarcoma compared with patients had pre-operative uterine sarcoma diagnosis $(42.8 \%$ vs $14.2 \%$, respectively, $\mathrm{p}=0.002)$. All patients with recurrent disease in unexpected sarcoma group had local recurrence $(100 \%)$. Regarding survival rates, there was no statistically difference between two groups ( $65.3 \%$ vs $57.1 \%)$.

Uterine sarcoma is a rare tumor entity. Unexpected sarcoma cases found to have high recurrence rate and had totally local metastases. This finding may result from "myomectomy procedure" itself.
\end{abstract}

Key Words: Uterine sarcoma, unexpected uterine sarcoma, leiomyosarcoma, myomectomy

\section{Introduction}

Uterine sarcomas are rare malignant tumors that account for $2-5 \%$ of all uterine malignancies (1). Despite intensive treatment, local recurrence and distant metastases are common. When a correct diagnosis is made preoperatively, surgical staging consisting of hysterectomy, salpingooopherectomy and lymph node resection is the mainstay of treatment (2). Addition of radiotherapy, chemotherapy, or hormonal therapy depends on the stage of the disease (3). Because it is not generally feasible to distinguish a sarcoma from a leiomyoma preoperatively, uterine sarcomas are often diagnosed only after a surgical procedure is performed for a mass that is presumed benign. When a uterine sarcoma is incidentally detected after myomectomy, there is concern that morcellation and/or myoma extraction could lead to unintended dissemination of malignant tissue throughout the peritoneum. Several retrospective series of patients with leiomyosarcoma reported worse outcomes in patients who had undergone previous morcellation (4-7). However, some studies have indicated poor outcomes among patients with unexpected leiomyosarcoma after morcellation as compared with those without morcellation due to its inherent poor prognosis $(5,8)$.

Given the low incidence, current data on uterine sarcomas depend on case reports and small retrospective case series. In the same time, few studies seem to have reported the risk and prognosis of unexpected uterine sarcoma after myomectomy and/or hysterectomy for presumed leiomyomas. Our aim was to study the cases of uterine sarcoma in the period from January 2009 to December 2016, regarding incidence, stage of disease at time of diagnosis, and survival rates. We also aimed to compare clinical outcome of preoperatively or postoperatively (unexpected) diagnosed uterine sarcoma cases.

\footnotetext{
${ }^{*}$ Corresponding Author: Cetin Kılıççı, Zeynep Kamil Maternity and Children’s Training and Research Hospital, Department of Obstetrics and Gynecology Üsküdar/İstanbul 34668 


\section{Materials and Methods}

We performed a retrospective descriptive review of patients with histologically proven uterine sarcoma at the Zeynep Kamil Women and Children Education and Research Hospital, Department of Obstetrics and Gynecology, a tertiary referral center, from January 2009 to December 2016. The study was approved by the ethic committee of the hospital, and verbal consent was obtained from all patients during the follow-up phone call.

Data were collected from medical records of patients. The age at diagnosis, gravidity, parity, menopausal status, complaints, comorbidity, tumor grade, tumor histology, stage of disease, postoperative adjuvant therapy, recurrence rate, location of recurrence, follow-up interval, and survival rate. All cases underwent laparotomy in myomectomy procedure and morcellation not applied to any case. Additionally, patients were divided into two groups: patients with diagnosed preoperatively and diagnosed postoperatively (unexpected). Two groups were compared regarding clinical characteristics.

The World Health Organization classification (2003) for uterine sarcomas and the International Gynecology and Obstetrics staging system (2009) were applied to all cases.

SPSS (Statistical Package for the Social Sciences) version 16.0 (SPSS Inc, Chicago, Ill) was used for statistical analyses. Descriptive statistics were used to describe the patients' characteristics. Two groups were compared using Fisher exact test for categorical variables. Two-sided values of $\mathrm{p}<0.05$ were considered statistically significant.

\section{Results}

Between January 2009 and December 2016, 63 patients were found to have uterine sarcoma. Mean age of patients was 51.8 \pm 11.7 , ranging from 23 to 74 years. Thirty-eight patients $(60.3 \%)$ were at menopause at time of diagnosis. The most common complaint was abnormal uterine bleeding $(n=33$, $52.4 \%)$ followed by pelvic pain $(n=19,30.1 \%)$ and pelvic mass $(\mathrm{n}=11,17.4 \%)$.

Histologic tumor type showed that $29(46 \%)$ patients had leiomyosarcoma, 11 (17.5\%) patients had endometrial stromal sarcoma and $13(20.6 \%)$ had malign mixt mullerian tumor. Other types were found as adenosarcoma $(n=4)$, carcinosarcoma $(n=4)$, fibrosarcoma $(\mathrm{n}=1)$, and liposarcoma $(\mathrm{n}=1)$. Thirtytwo $(50.8 \%)$ patients were low grade and $31(49.2 \%)$ patients were high grade.
In all, there were 37 patients with stage I disease, 7 with stage II, 12 with stage III and 7 patients with stage IV. Patients with disease confined to the uterus $(\mathrm{n}=15)$ received no adjuvant treatment $(23.8 \%)$. Twenty-five $(39.7 \%)$ patients received adjuvant radiotherapy and $24(38.1 \%)$ patients received adjuvant chemotherapy. Mean follow-up period was 35.1 \pm 20.7 months (range 4-84 months). Thirteen patients $(20.6 \%)$ had disease recurrence. Nine patients $(14.2 \%)$ had local recurrence while 4 patients $(6.3 \%)$ had distant metastases to the lungs. The overall survival rate was $63.5 \%(\mathrm{n}=40)$.

Of 63 patients, 49 had pre or intra operative uterine sarcoma diagnosis and 14 patients were incidentally found to have an unexpected uterine sarcoma after myomectomy by postoperative histology. Restaging operation was performed at these 14 patients. Of 49 patients, 30 had frozen section. A total of 4252 patients were identified as having undergone myomectomy or hysterectomy for presumed myomas during study period. The incidence of unexpected sarcoma was detected as $0.3 \%(14 / 4252)$. The comparison of patients with diagnosed preoperatively and diagnosed postoperatively (unexpected) were shown in Table I. There was no statistically significant difference regarding histologic type, grade, stage of disease, and adjuvant therapy. The recurrence rate was significantly higher in patients with unexpected uterine sarcoma compared with patients who had preoperative uterine sarcoma diagnosis $(42.8 \%$ vs $14.2 \%$, respectively, $\mathrm{p}=0.002)$. All patients with recurrent disease in unexpected sarcoma group had local recurrence $(100 \%)$. There were no patients with distant metastases in this group. Regarding survival rates, there was no statistically difference between two groups $(65.3 \%$ vs $57.1 \%)$.

\section{Discussion}

Uterine sarcoma is a rare tumor with high malignant potential and poor prognosis. Despite being derived from various cellular origins and gaving different pathologic features, most sarcomas have aggressive behaviour, high rates of recurrence and poor prognosis even when diagnosed at an early stage.

Our study found that leiomyosarcoma as the most common type of uterine sarcoma, followed by malign mixt mullerian tumor. Most studies of uterine sarcomas report an incidence of $55 \%$ for leiomyosarcoma, however, Sivakumari et al (1) reported that endometrial stromal sarcoma $(69 \%)$ is the commonest type of sarcoma in an Indian tertiary centre over 8 year period. On the other hand, Hosh et al (3) identified 13.089 patients diagnosed with uterine sarcoma between 2000-2012 in Egypt and they 
Table 1. Demographic characteristics of uterine sarcoma cases. LMS: Leiomyosarcoma, ESS: Endometrial stromal sarcoma, MMT: Malign mixed mullerian tumor

\begin{tabular}{|c|c|c|c|}
\hline & Group I & Group II & $\mathrm{p}$ \\
\hline & $\mathrm{n}(\%)$ & $\mathrm{n}(\%)$ & \\
\hline Age & $52.8 \pm 10.5$ & $48.4 \pm 15.0$ & 0.21 \\
\hline Gravidity & $4.54 \pm 2.79$ & $4.00 \pm 2.19$ & 0.52 \\
\hline Parity & $3.19 \pm 2.30$ & $2.92 \pm 1.44$ & 0.69 \\
\hline Menopause & $31(52.5)$ & $7(50)$ & 0.37 \\
\hline Complaint & & & 0.06 \\
\hline Pelvic pain & $12(24.4)$ & $7(50)$ & \\
\hline Pelvic mass & $11(22.4)$ & 0 & \\
\hline Abnormal bleeding & $26(53)$ & $7(50)$ & \\
\hline Comorbidity & $21(42.8)$ & $7(50)$ & 0.63 \\
\hline Histologic type & & & 0.21 \\
\hline LMS & $23(46.9)$ & $6(42.8)$ & \\
\hline ESS & $9(18.3)$ & $2(14.2)$ & \\
\hline MMT & $10(20.4)$ & $3(21.4)$ & \\
\hline Adenosarcoma & $3(6.1)$ & $1(7.1)$ & \\
\hline Carcinosarcoma & $4(8.1)$ & 0 & \\
\hline Fibrosarcoma & 0 & $1(7.1)$ & \\
\hline Liposarcoma & 0 & $1(7.1)$ & \\
\hline Grade & & & 0.50 \\
\hline Low-grade & $26(53)$ & $6(42.8)$ & \\
\hline High-grade & $23(46.9)$ & $8(57.1)$ & \\
\hline Stage & & & 0.09 \\
\hline Stage Ia & $10(20.4)$ & $5(35.7)$ & \\
\hline Stage Ib & $20(40.8)$ & $2(14.2)$ & \\
\hline Stage IIa & $2(4.08)$ & $3(21.4)$ & \\
\hline Stage IIb & $1(2.04)$ & $1(7.1)$ & \\
\hline Stage IIIc & $10(20.4)$ & $2(14.2)$ & \\
\hline Stage IVa & $5(10.2)$ & 0 & \\
\hline Stage IVb & $1(2.04)$ & $1(7.1)$ & \\
\hline Adjuvant radiotherapy & $21(42.8)$ & $4(28.5)$ & 0.33 \\
\hline Adjuvant chemotherapy & $16(32.6)$ & $8(57.1)$ & 0.09 \\
\hline Recurrence & & & 0.002 \\
\hline None & $42(85.7)$ & $8(57.1)$ & \\
\hline Local & $3(6.1)$ & $6(42.8)$ & \\
\hline Distant & $4(8.1)$ & 0 & \\
\hline Survival rate & $32(65.3)$ & $8(57.1)$ & 0.57 \\
\hline
\end{tabular}

reported that carcinosarcoma is the most common uterine sarcoma subtype as reported by a study from Thailand (2). These differences may be attributed to different age population, race and geographic characteristics.

Regarding symptoms of the patients with uterine sarcoma, previous studies reported $59-82 \%$ of patients had abnormal uterine bleeding and $17-23 \%$ presented with mass (2). In our study, the most common presenting symptom was abnormal uterine bleeding followed by pelvic pain.

Nearly $60 \%$ of our patients had early stage of disease. This was comparatively low compared to the other studies which reported $60 \%-76 \% \quad(9,10)$. The differences may be due to the extent of operative procedure including lymph node surgical evaluation. 
Our institution is a tertiary center for cancer care and almost all of the surgical procedures were performed by the gynecologic oncologist who generally performed complete surgical staging if the diagnosis of malignancy was made pre- or intra-operation.

Adjuvant chemotherapy and radiotherapy were almost equally given to patients, $38.1 \%$ and $39.7 \%$, respectively. Disease recurrence rate was low $(20.6 \%)$ compared with other studies which found recurrences ranging from $37 \%$ to $63 \%$ (2). Overall survival rate was found as $63.5 \%$ in our study. While Potikul et al reported that 2-year overall survival rate of uterine sarcoma cases was $49 \%$, Bostanci et al (11) reported that 12 of 29 uterine sarcoma cases were died with the average life expectancy of 3.2 years. The low recurrence rate and relatively high survival rates may be due to various prognostic factors found in each study. The patient characteristics (age, size of tumor, stage of disease, hystologic types, grade) as well as the treatment received (type of surgery, surgeon's skill for oncologic surgery, adjuvant therapy) may explain our low recurrence and high survival rates.

In our study, the incidence of unexpected uterine sarcoma was $0.3 \%$ as reported other studies $(12,13)$. When we compared clinical characteristics of preoperatively and postoperatively diagnosed uterine sarcoma cases, we found that recurrence rate was significantly higher in patients with unexpected uterine sarcoma. Regarding site of metastases, all patients with unexpected sarcoma had local recurrence. In our study, no incision was performed to the uterus in preoperatively diagnosed sarcoma cases. However, in unexpected uterin sarcoma cases a myometrial incision has already been performed. Our findings may result from presence of myometrial incision. At myomectomy procedure, myometrium is incised and leiomyoma is extracted aggressively via sharp clamps. This maneuver should carry a risk of tissue dissemination. However, survival rates of preoperatively and postoperatively diagnosed uterine sarcoma cases were comparable.

Patients had undergone unplanned excision of a soft tissue sarcoma before being referred to an oncology center had worse local control as reported by Gustafson et al (14). Perri et al (6) also reported that primary surgery involving tumor injury seems to be associated with a worse prognosis than total hysterectomy as primary intervention in 37 patients with stage I leiomyosarcoma. They did not mention about increased risk of local recurrence. We found increased risk of local metastasis accompany with comparable survival rate in unexpected uterine sarcoma cases after myomectomy.

Recently, there has been an increasing focus on the risk of spreading malignant tissues by laparoscopic power morcellation during laparoscopic myomectomy. The US Food and Drug Administration (FDA) discouraged the use of power morcellation for myomas in view of the potential risk, which might significantly worsen long-term survival (15). In a study, the prognosis of unexpected uterine sarcomas after laparoscopic (with power morcellation) and laparotomic (without power morcellation) myomectomy was compared (16). The authors found that all unexpected uterine sarcoma cases were alive after a mean follow-up of 31.2 months in the laparoscopy group and 40.5 months in the laparotomy group. One local recurrence was recorded in laparotomy group and this patient was also alive at 20 months after cytoreductive surgery. However, they had only 9 cases of unexpected uterine sarcoma, including 1 with leiomyosarcoma and 8 with an endometrial stromal sarcoma. This favorable prognosis may result from inherent good prognosis of endometrial stromal sarcoma. When the present study was considered together with this study, local recurrence of uterine sarcoma can be induced by myomectomy procedure itself regardless of laparoscopy or laparotomy. However, it is not clear that power morcellation brings more tissue dissemination risk in laparoscopic myomectomy for unexpected uterine sarcoma.

In conclusion, our study showed clinical characteristics and prognosis of preoperatively and postoperatively diagnosed uterine sarcoma cases performed by laparatomy. Unexpected sarcoma cases found to have high recurrence rate and had totally local metastases. This finding might be attributed to "myomectomy procedure" itself. We think that the prognosis of unexpected uterine sarcomas will be 'inherently same' regardless of laparoscopy or laparatomy. However, these results should be interpreted with caution. Further studies should be encouraged to evaluate the prognosis of unexpected uterine sarcomas operated by laparotomy or laparoscopy including power morcellation.

\section{Declaration of Interest: None}

\section{References}

1. Sivakumari S, Rajaraman R, Subbiah S. Uterine sarcoma: The Indian Scenario. Indian J Surg Oncol 2015; 6: 232-236.

2. Potikul C, Tangjitgamol S, Khunnarong J, et al. Uterine sarcoma: Clinical presentation, treatment and survival outcomes in Thailand. Asian Pac J Cancer Prev 2016; 17: 1759-1767.

3. Hosh M, Antar S, Nazal A, et al. Uterine sarcoma: Analysis 13.089 cases based on surveillance,

East J Med Volume:23, Number:2, April-June/2018 
epidemiology, and end results database. Int J Gyn Cancer 2016; 26: 1098-104.

4. George S, Barysauskas C, Serrano C, et al. REtrospective cohort study evaluating the impact of intraperitoneal morcellation on outcomes of localized uterine leiomyosarcoma. Cancer 2014; 120: 3154-3158.

5. Park JY, Park SK, Kim DY, et al. The impact of tumor morcellation during surgery on the prognosis of patients with apperently early uterine leiomyosarcoma. Gynecol Oncol 2011; 122: 255259.

6. Perri T, Korach J, Sadetzki S, et al. Uterine leiomyosarcoma: Does the primary surgical procedure matter? Int J Gyn Cancer 2009; 19: 257-260.

7. Morice P, Rodrigez A, Rey A, et al. Prognostic value of initial surgical procedure for patients with uterine sarcoma: Analysis of 123 patients. Eur J Gynecol Oncol 2003; 24: 237-240.

8. Seidman MA, Oduyebo T, Muto MG, et al. Peritoneal dissemination complicating morcellation of uterine mesenchymal neoplasms. PLoS One 2012; 7:e50058.

9. Benito V, Lubrano A, Arencibia O, et al. Clinopathologic analysis of uterine sarcomas from a single institution in the Canary Islands. Int J Gynaecol Obstet 2009; 1079: 44-49.

10. Durnali A, Tokoglu S, Ozdemir N, et al. Prognostic factors and treatment outcomes in 93 patients with uterine sarcoma from 4 centers in
Turkey. Asian Pac J Cancer Prev 2012; 13: 1935 1941.

11. Bostanci E, Ayas S,Gurbuz TA, et al. Retrospective analysis of 29 cases with uterine sarcoma: A single center experience. Dicle Medical Journal 2015; 42: 175-180.

12. Parker WH, Fu YS, Berek JS. Uterine sarcoma in patients operated on for presumed leiomyoma and rapidly growing leiomyoma. Obstet Gynecol 1994; 83: 414-418.

13. Sinha R, Hegde A, Mahajan C. et al. Laparoscopic myomectomy: do size, number, and location of the myomas from limiting factors for laparoscopic myomectomy? J Minim Invasive Gynecol 2008; 15: 292-300.

14. Gustafson P, Dreinhofer KE, Rydholm A. Soft tissue carcinoma should be treated at a tumor center. A comparison of quality of surgery in 375 patients. Acta Orthop Scand 1994; 65: 47-50.

15. US Food and Drug Administration. Quantitative Assessment of the prevalence of unsuspected uterine sarcoma in women undergoing treatment of uterine fibroids. Available at: http://www.fda.gov/downloads/medicaaldevices /safety/alert and notices/ucm393589.pdf. Accesssed April 17, 2014.

16. Zhang J, Zhang J, Dai Y, et al. Clinical characteristics and management experience of unexpected uterine sarcoma after myomectomy. Int J Gyn Obstet 2015; 130: 195-199. 